\title{
Language differences in qualitative research: is meaning lost in translation?
}

\author{
Fenna van Nes $\cdot$ Tineke Abma $\cdot$ Hans Jonsson • \\ Dorly Deeg
}

Published online: 19 November 2010

(c) The Author(s) 2010. This article is published with open access at Springerlink.com

\begin{abstract}
This article discusses challenges of language differences in qualitative research, when participants and the main researcher have the same non-English native language and the non-English data lead to an English publication. Challenges of translation are discussed from the perspective that interpretation of meaning is the core of qualitative research. As translation is also an interpretive act, meaning may get lost in the translation process. Recommendations are suggested, aiming to contribute to the best possible representation and understanding of the interpreted experiences of the participants and thereby to the validity of qualitative research.
\end{abstract}

Keywords Qualitative research - Methods - Language · Translation

Communicated by H.W. Wahl.

F. van Nes $(\varangle) \cdot$ D. Deeg

Longitudinal Aging Study Amsterdam, EMGO Institute

for Health and Care Research, VU University Medical Center,

Van der Boechorststraat 7, 1081 BT Amsterdam,

The Netherlands

e-mail: f.a.van.nes@hva.nl

F. van Nes

Amsterdam School of Health Professions, Amsterdam,

The Netherlands

T. Abma

Medical Humanities, VU University Medical Center,

Amsterdam, The Netherlands

H. Jonsson

Division of Occupational Therapy, Karolinska Institutet,

Stockholm, Sweden
English is the dominant language in cross-European projects and publications (Kushner 2003). With European research collaboration and knowledge circulation being stimulated by the European Union as well as by national governments, language differences play an increasingly important role in research. Language differences may have consequences, because concepts in one language may be understood differently in another language. This is in particular relevant for qualitative research, because it works with words; language is central in all phases ranging from data collection to analysis and representation of the textual data in publications. Language differences may occur in the first phase of a qualitative study, when interview data need to be translated to the researcher's language, for example in qualitative research with immigrants. Consequences for the validity of moving across languages have gained considerably attention in these cross-cultural studies (Squires 2009). However, language differences also play a role, when translation is required in later phases. This is the case in most studies with participants and main researcher having the same non-English native language, because publication is sought mainly in English outlets. In these studies, moving to English has gained little methodological attention, although here validity might be threatened as well. This article discusses challenges of language differences in qualitative research, when participants and the main researcher have the same non-English native language and the non-English data lead to an English publication.

\section{Interpretation of meanings}

Qualitative research seeks to study meanings in subjective experiences. The relation between subjective experience 
and language is a two-way process; language is used to express meaning, but the other way round, language influences how meaning is constructed. Giving words to experiences is a complicated process as the meaning of experiences is often not completely accessible for subjects and difficult to express in language. To capture the richness of experience in language, people commonly use narratives and metaphors (Polkinghorne 2005). Metaphors vary from culture to culture and are language-specific (Lakoff and Johnson 1980). For example in Dutch it is a common saying to give a proposal 'hands and feet' (handen en voeten geven in Dutch) to express the physical work that is needed to make the proposal concrete. This expression is not easily understandable for native English speakers (Otis 2008). Language also influences what can be expressed, and some linguists even state that social reality as experienced is unique to one's own language; those who speak different languages would perceive the world differently (Chapman 2006).

Qualitative research is considered valid when the distance between the meanings as experienced by the participants and the meanings as interpreted in the findings is as close as possible (Polkinghorne 2007). We would like to go one step further, and hold that the findings should be communicated in such a way that the reader of the publication understands the meaning as it was expressed in the findings, originating from data in the source language.

Translation between languages involves interpretation as well. The message communicated in the source language has to be interpreted by the translator (often the researcher him or herself) and transferred into the target language in such a way that the receiver of the message understands what was meant. Challenges in the interpretation and representation of meaning may be experienced in any communicative action, but are more complicated when cultural contexts differ and interlingual translation is required. Because interpretation and understanding meanings are central in qualitative research and text is the 'vehicle' with which meaning is ultimately transferred to the reader, language differences generate additional challenges that might hinder the transfer of meaning and might result in loss of meaning and thus loss of the validity of the qualitative study.

\section{Challenges of language differences}

We will now discuss the challenges that may arise when moving to English in qualitative research. We give examples to illustrate these challenges, although it is challenging in itself to formulate in English examples of the problems in translation between non-English to English. Where needed we have kept the original words in the source language.
Translation of findings

With participants and the main researcher speaking the same language, no language differences are present in data gathering, transcription and during the first analyses, because usually the first coding phase stays closely to the data. The first language differences may occur when interpretations are being discussed among members of a multinational research team. This is a fragile phase with multiple interpretations being under discussion as even in the source language it is not yet clear how to express the meanings as interpreted. For discussion, these first interpretations need to be explained in English and a very good understanding of subtle meaning differences is needed to come to the best English wordings. A first example comes from a study with ageing couples. The multinational team discussed how to express the particular way in which the couples experienced changes, namely as slow and almost unnoticed 'movements down a slope'. The words 'shifting' and 'gliding' were considered, but both words seemed not fully suitable to express the intended meaning.

In this example, the translation challenges occurred in the first interpretation phase. In the following example, we were not aware of translation problems when translating the Dutch wandelen to walking, because according to several dictionaries, 'walking' was linguistically correct. However, native English speakers understood walking as the Dutch lopen, as to move from one place to another on feet, only as instrumental transportation. However, the activity wandelen consisted of a complex constellation of different meanings including the intrinsic enjoyment of the activity, enjoying nature and its associations with Sunday afternoons and holidays together. Ultimately 'going for walk' seemed more appropriate to represent the meaning expressed by the couple.

Challenges of translation may even occur when support of a professional translator is been used. This occurred in a narrative case study of an older couple after the wife had experienced a stroke (Van Nes et al. 2009). The findings had the form of narratives with the main meanings expressed in the titles of the narratives. The common narrative was that they acted as one organism, which was expressed as 'One body, three hands and two minds'. The title of the narrative of the husband was constructed to express the sudden and complete shift in his valued activities. Before the stroke of his wife, he had his own engaging activities (bee-keeping and having a kitchen garden), which he experienced as a way to be independent and to support himself and the household with honey and vegetables. After the stroke of his wife, he was busy all day long with supporting her and there was no time left for these former valued activities. In the title 'From being 
self-supporting in an engaging occupation ${ }^{1}$ to the absorbing occupation of supporting', the word engaging was meant to reflect a positive meaning. Absorbing was meant to contrast with engaging and to indicate that he was fully occupied all day long after the stroke. The reviewers of the submitted paper on this study, however, understood both English words as having the same positive connotation, so the intended meaning of the complete turn was lost.

\section{Translation of quotations}

Quotations of participants are commonly being used in qualitative research articles. Translation of quotes poses specific challenges, because it may be difficult to translate concepts for which specific culturally bound words were used by the participants. For example, the Dutch word gezellig was used commonly by late-life couples, expressing the feeling they had when doing things together. The meaning expressed with this typical Dutch word included experiencing togetherness in doing everyday activities together, often at specific times of the day and in the own home. Translating the word gezellig, only as 'cosy' would reduce the meaning. Using more words than in the original quote, however, changes the voice of the participant. This is especially problematic as giving voice to people is seen as an important aim of qualitative research (Denzin and Lincoln 2000).

\section{Back translation}

After publication, a new translation challenge may be faced, when back translation to the original source language is undertaken. This was the case when translating 'One body, three hands and two minds' back to Dutch. The literal translation of 'One body' would have been 'Eén lichaam', but this appeared to be more physical than one body, because in English the word body is also used in other ways, e.g. as in a body of literature. The chosen solution was translation as Samen Eén ('Together One'), but here the meaning of functioning as one organism was lost.

We have shown that with interpretation of meaning being central in qualitative research, language differences may affect the understanding and interpretation of meanings in different phases on the way from participant to reader. If translation issues are not given adequate thought and attention, the meaning-transfer-chain may resemble the whispering game children play. In the game players line up in such a way that they can whisper to their immediate neighbours. The first player whispers a phrase to his neighbour, who then passes on the message until it reaches

\footnotetext{
${ }^{1}$ Occupation is used in Occupational Science as a broad construct as "meaningful everyday activities".
}

the end of the line. If the game has been 'successful', the final message differs considerably from the first. In qualitative research meaning is also transferred from one phase to the next, until it reaches the reader and in each transfer meaning might get lost. Such loss of meaning reduces the validity of the qualitative study.

\section{Recommendations}

In the following, we give some recommendations aimed to potentially reduce the loss of meaning and thereby to enhance the validity of cross-English qualitative research. Our first recommendation focuses on the thinking and reflection processes that are needed in the analyses. We experienced that talking and reading in English leads to thinking in the English language as well. The relationship between thinking and language has been studied from different scientific perspectives, e.g. in psychology and in the philosophy of language (see e.g. Jackendoff 2009). One view considers language to be an aid to thinking. It is beyond the scope of this article to examine this relationship further. However, we feel it can be stated that there is some influence when analysing in another language than your own. To avoid potential limitations in the analysis we therefore recommend staying in the original language as long and as much as possible.

In discussions with members of the research team or peers who do not speak the source language, we recommend to delay the use of fixed-one word-translations. Instead, the analyses might even benefit from using fluid descriptions of meanings using various English formulations. In doing so, it is important to check the interpretations by going back to the codes and preliminary findings in the source language. Keeping record of these discussions would be useful to make the development of the interpretations transparent when in later phases the translations need to be adapted.

For translation of the most meaningful language parts in the findings, like the titles in narrative research or the themes in phenomenological research, we recommend that the researcher operates as a translation moderator in cooperation with a professional translator. This would involve explaining to the translator the intended meaning and its context in the source language. We recommend this should be done in a side-by-side procedure, in which the researcher and the translator discuss possible wordings. Often, different linguistically correct translations are possible, but there will be subtle meaning differences, which need to be closely examined in order to decide on the best translation.

Rich descriptions with the use of quotes of participants are considered to contribute to trustworthiness in qualitative 
research. However, using quotes is not unproblematic, because participants might feel that they are not fairly represented, when they see their spoken words in written form. Translating the quotes to another language enlarges this problem, because in the translation the words are literally not their own anymore (Temple 2008). Therefore, we recommend that these translations are also undertaken with support of a professional translator. Special attention is needed when metaphors are translated, either in quotes or in the findings.

Currently, in method sections of English articles reporting research with non-English data, translation issues are seldom discussed. In line with cross-language research literature, we recommend to describe and discuss in the research article how translation has been undertaken. This will provide reviewers and readers alike with a better insight into the way potential meaning losses have been avoided in the procedures used (Squires 2009).

We are aware that using the services of a professional translator adds to the costs of a study. However, these costs contribute to improving the validity of the research and of the quality of the transference of the findings to the readers of the publication. Furthermore, we suggest that the use of a translator in earlier phases of the research reduces efforts to refine translations in later phases, and may prove to be enriching as the discussing about the best translation may reveal new layers of meanings.

The recommendations we presented here are formulated for qualitative researchers who present findings in English, while the data were gathered in their native non-English language. For cross-European research in general, we consider some of our recommendations also to be relevant. In particular, the recommendation to use fluid descriptions of meanings in discussions might enhance quality also in quantitative studies, because it contributes to a sound understanding among researchers of the concepts central to their research.

We consider these recommendations to be a first step, since more research is needed on this topic. First, we would recommend to undertake an inventory with a questionnaire among non-English researchers who published their qualitative research in English outlets in order to collect data how they handled language differences in their studies and to get insight in and rise their awareness of the potential threat to validity when meaning gets lost in translation. As a next step, a series of focus groups could result in guidelines for cross-English qualitative research. With these suggestions for further steps, we stress the importance of an ongoing dialogue regarding different aspects of translation as an important methodological issue for qualitative cross-English research.

Open Access This article is distributed under the terms of the Creative Commons Attribution Noncommercial License which permits any noncommercial use, distribution, and reproduction in any medium, provided the original author(s) and source are credited.

\section{References}

Chapman S (2006) Thinking about language; Theories of English. Palgrave Macmillan, New York

Denzin NK, Lincoln YS (2000) Handbook of qualitative research, 2nd edn. Sage Publications, Thousand Oaks, CA

Jackendoff R (2009) Language, consciousness, culture: essays on mental structure. MIT Press, Cambridge

Kushner E (2003) English as global language: problems, dangers, opportunities. Diogenes 50:17-23

Lakoff G, Johnson M (1980) Metaphors we live by. The University of Chicago Press, Chicago

Otis L (2008) Going with your gut: some thoughts on language and the body. Perspect 372:798-799

Polkinghorne D (2005) Language and meaning: data collection in qualitative research. J Counsel Psychol 52:137-145

Polkinghorne D (2007) Validity issues in narrative research. Qual Inq 13:471-478

Squires A (2009) Methodological challenges in cross-cultural qualitative research: a research review. Int J Nurs Stud 46:277-287

Temple B (2008) Narrative analysis of written texts: reflexivity in cross language research. Qual Res 8:355-365

Van Nes F, Runge U, Jonsson H (2009) One body, three hands and two minds: a case study of the intertwined occupations of an older couple after a stroke. J Occup Sci 16:194-202 